\title{
Lung tissue gene-expression signature for the ageing lung in COPD
}

\author{
Maaike de Vries, ${ }^{1,2,3}$ Alen Faiz, ${ }^{1,3,4}$ Roy R Woldhuis, ${ }^{1,3}$ Dirkje S Postma, ${ }^{3,4}$ \\ Tristan V de Jong, ${ }^{3,5}$ Don D Sin, ${ }^{6,7}$ Yohan Bossé, ${ }^{8,9}$ David C Nickle, ${ }^{10}$ Victor Guryev, ${ }^{3,5}$ \\ Wim Timens, ${ }^{1,3}$ Maarten van den Berge, ${ }^{3,4}$ Corry-Anke Brandsma ${ }^{1,3}$
}

- Additional material is published online only. To view please visit the journal online (http://dx.doi.org/10.1136/ thoraxjn-2017-210074)

For numbered affiliations see end of article.

\section{Correspondence to} Dr Maaike de Vries, Department of Epidemiology, University Medical Center Groningen, Hanzeplein, $9713 \mathrm{GZ}$, The Netherlands:

m.de.vries04@umcg.nl

$\mathrm{MB}$ and $\mathrm{C}-\mathrm{AB}$ contributed equally.

Received 31 January 2017 Revised 1 November 2017 Accepted 6 November 2017 Published Online First 6 December 2017

\section{Linked}

- http://dx.doi.org/10.1136/ thoraxjnl-2017-211173

Check for updates

To cite: de Vries M, Faiz A Woldhuis RR, et al. Thorax 2018;73:609-617.

\section{ABSTRACT}

Introduction COPD is a chronic, progressive, inflammatory disease of the lungs and the third leading cause of death worldwide. The current knowledge of the pathophysiology of COPD is limited and novel insights in underlying disease mechanisms are urgently needed. Since there are clear parallels between ageing and COPD, we investigated genes underlying lung ageing in general and abnormal lung ageing in COPD.

Methods Whole genome mRNA profiling was performed on lung tissue samples ( $n=1197)$ and differential gene expression with increasing age was analysed using an adjusted linear regression model. Subsequent pathway analysis was performed using GeneNetwork and the gene-expression signature was compared with lung ageing in the Genotype-Tissue Expression (GTEx) project. In a subset of patients with COPD ( $n=311)$ and non-COPD controls $(n=270)$, we performed an interaction analysis between age and COPD to identify genes differentially expressed with age in COPD compared with controls, followed by gene set enrichment pathway analysis.

Results We identified a strong gene-expression signature for lung ageing with 3509 differentially expressed genes, of which $33.5 \%$ were found nominal significant in the GTEx project. Interestingly, we found $E D A 2 R$ as a strong candidate gene for lung ageing. The age ${ }^{*}$ COPD interaction analysis revealed 69 genes significantly differentially expressed with age between COPD and controls.

Conclusions Our study indicates that processes related to lung development, cell-cell contacts, calcium signalling and immune responses are involved in lung ageing in general. Pathways related to extracellular matrix, mammalian target of rapamycin signalling, splicing of introns and exons and the ribosome complex are proposed to be involved in abnormal lung ageing in COPD.

\section{INTRODUCTION}

COPD is a chronic and progressive, inflammatory lung disease, characterised by persistent airflow limitation. ${ }^{1}$ Although exposure to cigarette smoke is the main risk factor for COPD, its development also involves other environmental and occupational exposures. ${ }^{2}$ Patients with COPD suffer from severe respiratory symptoms, resulting in inability to work, invalidity and an overall worse quality of life. The prevalence of COPD is high, especially in the elderly, and will increase even further due to

\section{Key messages}

What is the key question?

- Can we identify a lung tissue gene-expression signature that reflects lung ageing and abnormal lung ageing in COPD?

What is the bottom line?

- We identified a strong gene-expression signature for lung ageing with $E D A 2 R$ as a strong candidate gene for lung ageing and found that genes differentially expressed with increasing age between patients with COPD and non-COPD controls are involved in processes related to extracellular matrix organisation, mammalian target of rapamycin signalling, splicing of introns and exons, and the ribosome complex.

Why read on?

- The data presented in this study enhances the current understanding of abnormal lung ageing in COPD by complementing the current data with genome-wide gene expression data specifically on lung tissue and provide several new candidate genes involved in lung ageing and abnormal lung ageing in COPD.

the ageing population, leading to a considerable economic and social burden. ${ }^{1}$ The current knowledge of the pathophysiology of COPD is limited and novel insights in the disease mechanisms are urgently needed.

A novel and interesting approach towards a better understanding of COPD is the accelerated lung ageing hypothesis. ${ }^{3}$ It has been postulated that acceleration of the normal ageing process is involved in the disease pathogenesis of several chronic degenerative diseases, including COPD. ${ }^{4}$ Ageing is defined by the progressive loss of physiological integrity, resulting in impaired function and increased risk of disease or death. ${ }^{35}$ While the normal ageing lung is characterised by a decline in lung function, loss of elasticity, alveolar enlargement and inflammation, the majority of these features are also observed in the lungs of patients with COPD. ${ }^{4}$ Moreover, several other markers of ageing such as telomere shortening, cellular senescence and stem cell exhaustion have shown to be increased in patients with COPD. ${ }^{6}$ Shortening of 
telomere length, as well as increased expression of the senescence marker $\mathrm{p} 21$ was demonstrated in circulating lymphocytes of patients with COPD. ${ }^{7-9}$ Furthermore, pulmonary fibroblasts of patients with COPD showed increased expression of senescence associated beta-galactosidase activity when compared with control fibroblasts. ${ }^{10}$ Thus, there are clear parallels between ageing and COPD and better understanding of the underlying mechanisms of lung ageing and accelerated lung ageing in COPD may provide highly warranted new insights into the pathogenesis of COPD.

Notwithstanding the fact that the evidence for a role of lung ageing in COPD is increasing, little is known about the genes underpinning lung ageing and accelerated lung ageing in COPD. Therefore, in this cross-sectional study we first investigated differences in gene expression associated with age in human lung tissue. Second, we generated a gene-expression lung tissue signature for accelerated lung ageing in subjects with COPD.

\section{Material and methods Lung tissue collection}

Lung tissue was collected from patients undergoing lung resection, lung volume reduction or transplant surgery at one of the three participating sites in Groningen (The Netherlands), Québec (Canada) or Vancouver (Canada) in accordance with local ethical guidelines as previously described. ${ }^{11}{ }^{12}$ If resection surgery was conducted for tumour removal, macroscopically normal lung tissue was taken far distant from the tumour. All samples were histologically checked for abnormalities using standard H\&E staining.

For the gene-expression signature for lung ageing, we included a total of 1197 subjects from the database. The subjects were between 4 years and 85 years of age and subjects with unknown age were excluded as well as subjects with cystic fibrosis. For the age*COPD interaction analysis, a selection of 311 well defined current and ex-smoking ( $>5$ pack-years) patients with COPD $\left(\mathrm{FEV}_{1} / \mathrm{FVC}\right.$ ratio $\left.<70 \%\right)$ and 270 non-COPD controls $\left(\mathrm{FEV}_{1} /\right.$ FVC ratio $>70 \%$ ) was used. ${ }^{12}$

\section{Gene expression arrays}

Total RNA was extracted from lung tissue at Rosetta Inpharmatics Gene Expression Laboratory (Seattle, Washington, USA) for all three centres. mRNA profiling was performed using a custom-made Affymetrix HU133 array (GPL 10379) containing 751 controls probe sets and 51627 non-control probe sets. Unfiltered gene expression was normalised with the Robust Multichip Average method with background subtraction implemented in the Affymetrix Power Tools software V.1.8.5. ${ }^{11}$ For the analyses, the $\log 2$ (microarray intensity) values of the expression are used. The data are deposited at the Gene Expression Omnibus of the National Centre for Biotechnology Information with the accession number GSE23546.

\section{Statistics}

Linear regression analyses were performed using $\mathrm{R}$ software V.3.2.3. ${ }^{13}$ To adjust for strong expression differences between the samples due to unknown factors such as batch or technical variation, we used the common principal component approach. ${ }^{11} 12$ We calculated the principal components for each cohort separately and adjusted all our analyses for the principal components explaining more than $1 \%$ of the variation within each cohort. The potential confounders age, gender, smoking status and other diseases were excluded from the principal components calculation.
For the gene-expression signature for lung ageing, we additionally corrected for the potential confounders gender, smoking status (ie, never-smoking, current-smoking or ex-smoking) and disease if applicable (ie, COPD, non-COPD, unclassifiable COPD or non-COPD, alpha-1-antitrypsine deficiency, surgery for cancer, idiopathic pulmonary fibrosis, pulmonary hypertension or other diseases). For the age ${ }^{*} \mathrm{COPD}$ interaction analysis, we performed an age*COPD interaction analysis on the subset of the data set only including COPD and non-COPD controls on the three cohorts separately. This analysis was corrected for the potential confounders gender and smoking status (ie, currentsmoking vs ex-smoking) and technical variance as described before. Equations for all analyses are written down in the online Supplement.

For both of the analyses, we analysed the three cohorts separately and performed meta-analyses to identify genes that were differentially expressed with increasing age and behaved in the same direction across the three cohorts. To correct for multiple testing, the Benjamini-Hochberg false discovery rate (FDR) was applied.

\section{Sensitivity analyses}

The robustness of the gene-expression signature for lung ageing was tested in two subset analyses, that is, patients with COPD $(n=311)$ and non-COPD controls $(n=270)$. We compared the probe sets significantly associated with increasing age at nominal $\mathrm{P}$ value $<0.05$ in the same direction in the COPD and non-COPD control subsets with the 5732 probe sets significantly associated with increasing age in the whole data set.

To validate the gene-expression signature for lung ageing, we compared our signature with the lung ageing gene-expression signature identified in the Genotype-Tissue Expression (GTEx) project. ${ }^{14}$ Within this project, gene-expression signature in lung tissue was identified by modelling gene expression in a linear regression model using lung tissue of 119 subjects. From our differentially expressed genes (DEGs) with increasing age, ageing coefficients and $\mathrm{P}$ values of 3301 of these genes were available and the overlap in the same direction at nominal $\mathrm{P}$ value $<0.05$ was determined. In addition, we compared the associations between gene expression and age in our expression quantitative trait loci (eQTL) lung tissue database with adipose, artery, heart, lung, muscle, nerve, skin and thyroid tissues and blood from the GTEx for the top three most significant upregulated and downregulated genes with increasing age. Here, we used the age coefficient and the nominal $\mathrm{P}$ values for all the separate tissues as presented in the online supplement of the paper by Yang et al. ${ }^{14}$

The expression of $E D A 2 R$ was confirmed in lung tissue from 21 ex-smoking non-COPD control subjects using quantitative PCR analysis.

\section{GeneNetwork analysis}

For the gene-expression signature for lung ageing, we used GeneNetwork, in which the biological processes are ranked with principal component analyses, to predict the enrichment of biological processes of the top $10 \%$ of the DEGs. ${ }^{15}$

\section{Gene set enrichment analysis}

For age*COPD interaction analysis we performed functional enrichment analysis with gene set enrichment analysis (GSEA) (V.7.0.670.1). Genes were ranked according to the strength of their $\mathrm{t}$-statistics reflecting their interaction between age and COPD and subsequently analysed for enrichment in biological 


\begin{tabular}{|c|c|c|c|}
\hline & Groningen & Quebec & Vancouver \\
\hline Number & 357 & 470 & 370 \\
\hline Age, years (range)* & $56(6-83)$ & $64(30-84)$ & $63(4-85)$ \\
\hline Male/female, N & $187 / 170$ & $260 / 210$ & 197/173 \\
\hline \multicolumn{4}{|l|}{ Smoking, $\mathrm{N}^{*}$} \\
\hline Never & 74 & 46 & 37 \\
\hline Ex & 208 & 323 & 171 \\
\hline Current & 63 & 101 & 107 \\
\hline Unknown & 12 & 0 & 55 \\
\hline Pack-years, $\mathrm{N}^{*}$ & $\begin{array}{l}22 \\
(3-38)\end{array}$ & $\begin{array}{l}40 \\
(25-55)\end{array}$ & $\begin{array}{l}38 \\
(18-50)\end{array}$ \\
\hline $\mathrm{FEV}_{1}, \%$ predicted ${ }^{*}$ & $\begin{array}{l}38.9 \\
(20.5-77.0)\end{array}$ & $\begin{array}{l}82.4 \\
(70.6-94.6)\end{array}$ & $\begin{array}{l}85.4 \\
(70.0-98.3)\end{array}$ \\
\hline FVC, \% predicted ${ }^{*}$ & $\begin{array}{l}68.2 \\
(46.9-88.7)\end{array}$ & $\begin{array}{l}91.2 \\
(82.1-102.1)\end{array}$ & $\begin{array}{l}92.1 \\
(80.2-102.9)\end{array}$ \\
\hline $\mathrm{FEV}_{1} / \mathrm{FVC}, \% *$ & $\begin{array}{l}57.0 \\
(32.2-73.8)\end{array}$ & $\begin{array}{l}68.9 \\
(62.4-74.4)\end{array}$ & $\begin{array}{l}70.3 \\
(63.0-76.9)\end{array}$ \\
\hline Cancer surgery, \%* & 37.5 & 99.8 & 81.9 \\
\hline
\end{tabular}

Data are presented as medians with IQRs, unless otherwise stated.

*Significant differences among groups at $\mathrm{P}<0.05$ level $\left(\chi^{2}\right.$ and Kruskal-Wallis tests were applied to categorical and quantitative traits, respectively).

$\mathrm{FEV}_{1}$ : forced expiratory volume in one second , FVC: forced vital capacity .

pathways listed in the Kyoto encyclopaedia of genes and genomes (KEGG).

\section{Gene function prediction}

To predict the function of the genes highlighted in the text, we used a combination of GeneNetwork and Genecards (www. genecards.org). GeneNetwork is built on an independent gene expression data set of 77840 samples and predicts the function of genes based on known biological pathways available in the molecular signatures database MSigDB (http://www.broadinstitute.org). ${ }^{15}$

\section{Results}

\section{Subject characteristics}

An overview of demographical and clinical characteristics of the subjects included in this study are presented in table 1. A total of 1197 subjects were included in our analysis, with 357, 470 and 370 subjects in the Groningen, Quebec and Vancouver cohorts, respectively.

While there was a large range in age in all three cohorts, the median age of the cohorts was significantly different, possibly as a consequence of the lower age of the subjects in the Groningen cohort.

We did observe differences in smoking status and pack-years among the cohorts, with relatively more never smokers and lower number of pack-years in the Groningen cohort. Furthermore, the subjects from the Groningen cohort had a lower median lung function compared with the subjects in the cohorts from Quebec and Vancouver, and the above can be attributed to the fact that the Groningen cohort contained more subjects with very severe COPD undergoing lung transplantation. Based on the phenotypical differences between the cohorts described above, we analysed the cohorts separately, followed by a meta-analysis.

\section{Gene-expression signature for lung ageing}

Linear regression demonstrated a strong impact of age on lung tissue gene expression levels. Therefore, we restricted the FDR cut-off to $<0.0001$ and identified 5732 probe sets coding for 3509 significantly DEGs with age. With increasing age, 1980 genes were higher expressed, while 1529 genes were lower expressed with increasing age. Ectodysplasin A2 receptor (EDA2R), involved in the DNA damage response, was most significantly upregulated with age (FDR: $4.55 \times 10^{-63}$ ), while microsomal glutathione S-transferase 1 (MGST1), involved in detoxification and the defence response to oxidative stress, was most significantly downregulated with age (FDR: $8.59 \times 10^{-42}$ ). A complete list of these DEGs is provided in online supplementary table S1. A heatmap illustrating these results with the subjects ordered for each cohort separately based on their individual age is shown in figure 1A. To illustrate the expression pattern of the most significant DEGs with age, the individual gene expression of the top three genes most significantly upregulated $(E D A 2 R$, MAP4K1 and FRZB) and downregulated (MGST1, ZNF518B and $A T P 8 A 1)$ with age are shown in figure $1 \mathrm{~B}-\mathrm{D}$ and figure $1 \mathrm{E}-\mathrm{G}$, respectively.

Next, we performed pathway analysis using GeneNetwork ${ }^{15}$ to identify enriched biological processes to get more insight in the relevance of the DEGs to the ageing individual. To this end, we selected the top 10\% DEGs upregulated and downregulated with age. Genes upregulated with age were enriched for biological processes related to calcium signalling and immune responses, whereas genes downregulated with age were enriched for biological processes related to lung development and cellcell contacts. An overview of the 25 most enriched biological processes for DEGs altered with age is shown in table 2, the complete list of all the enriched biological pathways is presented in online supplementary table S2.

Taken together, by the identification of 3509 DEGs with increasing age, we show a strong gene-expression signature for the ageing lung.

\section{Sensitivity analysis of the lung ageing gene expression and} validation of EDA2R

To assess if the gene-expression signature for lung ageing was robust, we performed a sensitivity analysis in the selected COPD and non-COPD control subjects. Of the 5732 probe sets significantly associated with increasing age in the whole data set, we found an overlap at nominal P-value $<0.05$ of $70 \%$ and $72 \%$, respectively, in the COPD and non-COPD control subjects. A schematic overview of the comparison can be found in online supplementary figure S1a. Since the overlap is considerably higher than what can be expected by chance $(2.5 \%)$, we are confident that our gene-expression signature of the ageing lung is robust.

To further validate our gene-expression signature of the ageing lung, we determined the overlap with the lung ageing gene-expression signature identified in the GTEx project. ${ }^{14}$ From our signature, 3310 genes were present in the GTEx projects. By comparing these genes at nominal $\mathrm{P}$ value $<0.05$, we found an overlap of $33.5 \%$ in the same direction, which is 13 times more than what can be expected by chance. A schematic overview of the comparison can be found in online supplementary figure S1b.

Of interest, the gene most upregulated with age in our analysis, $E D A 2 R$ (figure $1 \mathrm{~B}$ ), was the second highest ranked gene among the genes upregulated with age in the GTEx project. Furthermore, in the sensitivity analyses in COPD and non-COPD 
A

GRONINGEN

QUEBEC

VANCOUVER

Change in gene expression

(z-score)

B

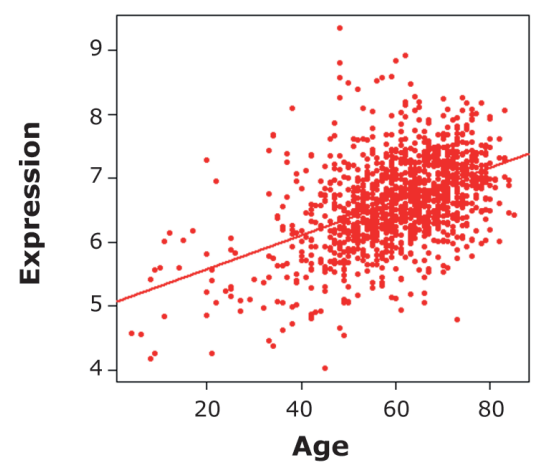

$\mathbf{E}$

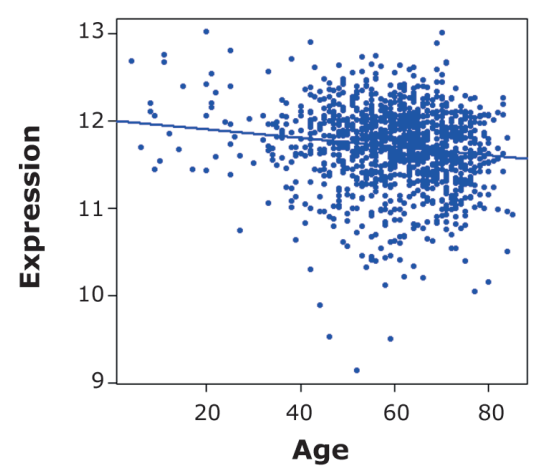

C

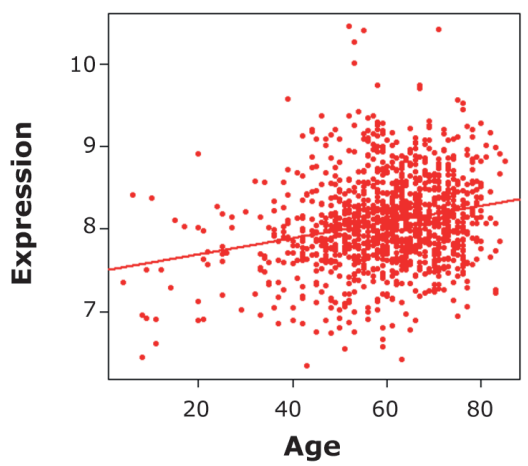

$\mathbf{F}$

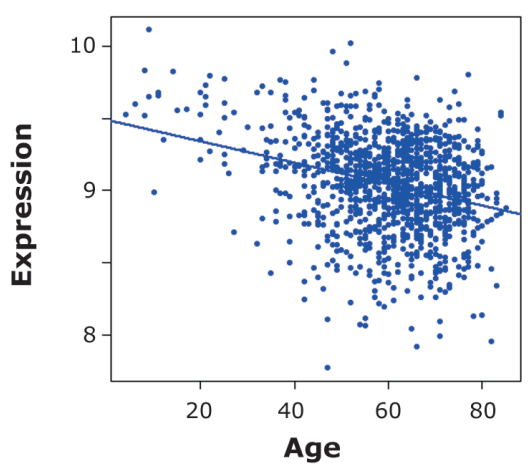

D

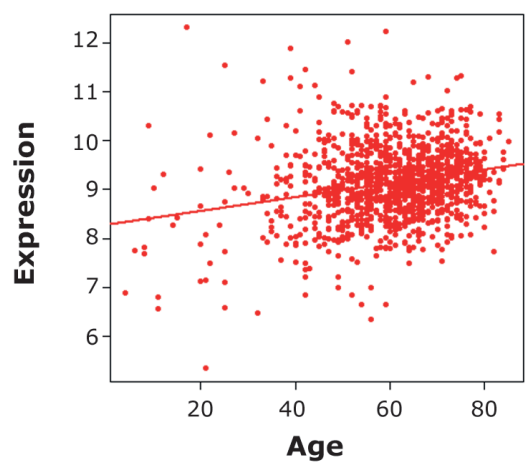

G

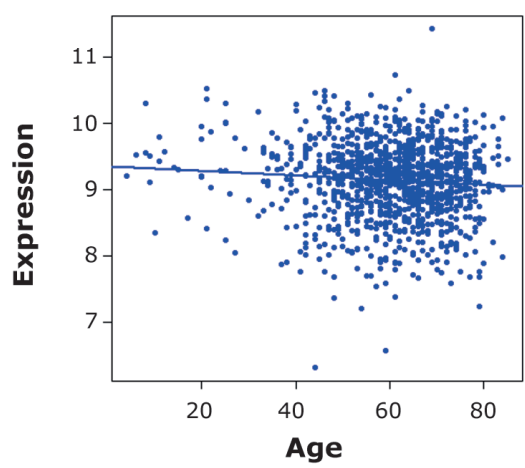

Figure 1 Differentially expressed genes upon increasing age in lung tissue. The heatmap shows probe sets coding for genes differentially expressed with increasing age at FDR $<0.0001$, for all the three cohorts from Groningen, Quebec and Vancouver. Each probe set is shown separately and for several genes more than one probe set was found to be significantly different. Relative increased gene expression is indicated in red, while relative decreased gene expression is indicated in blue. The top five genes upregulated and downregulated with increasing age are shown in the table next to the heatmap (1A). Log2 fold expression of the individual top three genes EDA2R, MAP4K1 and FRZB upregulated with increasing age (figure 1B-D). Log2 fold expression of the individual top three genes MGST1, ZNF518B and ATP8A1 downregulated with increasing age (figure 1E-G).

subjects, $E D A 2 R$ was the third highest ranked gene among the genes upregulated with age in both analyses. Moreover, quantitavie real time (qRT)-PCR validation of EDA2R in lung tissue of 21 ex-smoking non-COPD control subjects showed a significant association between increasing age and increased gene expression of $E D A 2 R$ (figure 2). Based on these four analyses, $E D A 2 R$ might be considered as a strong candidate gene for lung ageing in general.

To further confirm our findings in the independent GTEx data set and to assess whether the associations between 
Table 2 Top 25 enriched biological processes

\begin{tabular}{|c|c|c|c|}
\hline \multicolumn{2}{|l|}{ Genes decreased expressed with age } & \multicolumn{2}{|l|}{ Genes increased expressed with age } \\
\hline Biological processes & & Biological processes & \\
\hline Term & $P$ value & Term & $P$ value \\
\hline Lung alveolus development & $6 \times 10^{-17}$ & Cytosolic calcium ion transport & $2 \times 10^{-61}$ \\
\hline Tight junction assembly & $7 \times 10^{-16}$ & Release of sequestered calcium ion into cytosol & $3 \times 10^{-59}$ \\
\hline Apical junction assembly & $9 \times 10^{-16}$ & Regulation of sequestering of calcium ion & $3 \times 10^{-59}$ \\
\hline Lung development & $8 \times 10^{-14}$ & Negative regulation of sequestering of calcium ion & $3 \times 10^{-59}$ \\
\hline Respiratory tube development & $1 \times 10^{-13}$ & Calcium ion transport into cytosol & $3 \times 10^{-58}$ \\
\hline Respiratory system development & $5 \times 10^{-13}$ & Positive regulation of cell adhesion & $2 \times 10^{-49}$ \\
\hline Lung epithelium development & $4 \times 10^{-12}$ & Sequestering of calcium ion & $7 \times 10^{-47}$ \\
\hline Regulation of calcium-mediated signalling & $5 \times 10^{-12}$ & Homotypical cell-cell adhesion & $2 \times 10^{-44}$ \\
\hline Response to interleukin 6 & $5 \times 10^{-12}$ & Elevation of cytosolic calcium ion concentration & $5 \times 10^{-44}$ \\
\hline Epithelial tube branching involved in lung morphogenesis & $8 \times 10^{-12}$ & Cytosolic calcium ion homoeostasis & $5 \times 10^{-43}$ \\
\hline Asymmetrical protein localisation & $2 \times 10^{-11}$ & Regulation of cell adhesion & $5 \times 10^{-43}$ \\
\hline Lung cell differentiation & $2 \times 10^{-11}$ & Cellular calcium ion homoeostasis & $2 \times 10^{-42}$ \\
\hline Apical protein localisation & $2 \times 10^{-11}$ & Cellular divalent inorganic cation homoeostasis & $3 \times 10^{-41}$ \\
\hline Organophosphate metabolic process & $3 \times 10^{-11}$ & Regulation of neutrophil chemotaxis & $3 \times 10^{-40}$ \\
\hline Glycerolipid biosynthetic process & $6 \times 10^{-11}$ & Negative regulation of immune system process & $5 \times 10^{-40}$ \\
\hline Early endosome to late endosome transport & $7 \times 10^{-11}$ & Regulation of leucocyte migration & $1 \times 10^{-39}$ \\
\hline Lung epithelial cell differentiation & $9 \times 10^{-11}$ & Regulation of leucocyte chemotaxis & $2 \times 10^{-39}$ \\
\hline Phospholipid metabolic process & $9 \times 10^{-11}$ & Regulation of calcium-mediated signalling & $4 \times 10^{-39}$ \\
\hline Positive regulation of calcium-mediated signalling & $1 \times 10^{-10}$ & Calcium ion homoeostasis & $8 \times 10^{-39}$ \\
\hline Cell-cell junction assembly & $1 \times 10^{-10}$ & Calcium ion transport & $2 \times 10^{-38}$ \\
\hline Tube development & $2 \times 10^{-10}$ & Regulation of ion homoeostasis & $2 \times 10^{-38}$ \\
\hline Cell-cell junction organisation & $2 \times 10^{-10}$ & Sequestering of metal ion & $2 \times 10^{-38}$ \\
\hline Purine nucleoside triphosphate biosynthetic process & $2 \times 10^{-10}$ & Integrin-mediated signalling pathway & $4 \times 10^{-38}$ \\
\hline Regulation of nitric-oxide synthase activity & $2 \times 10^{-10}$ & Regulation of behaviour & $6 \times 10^{-38}$ \\
\hline Nucleoside triphosphate biosynthetic process & $2 \times 10^{-10}$ & Positive regulation of calcium-mediated signalling & $8 \times 10^{-38}$ \\
\hline
\end{tabular}

gene expression and age are specific for lung tissue, we compared the three most significant upregulated and downregulated genes with age from our lung tissue gene-expression signature with the available tissues within the GTEx project (online supplementary figure S2). The expression of the six genes was significantly associated with age in the same direction in lung tissue in the GTEx

\section{EDA2R}

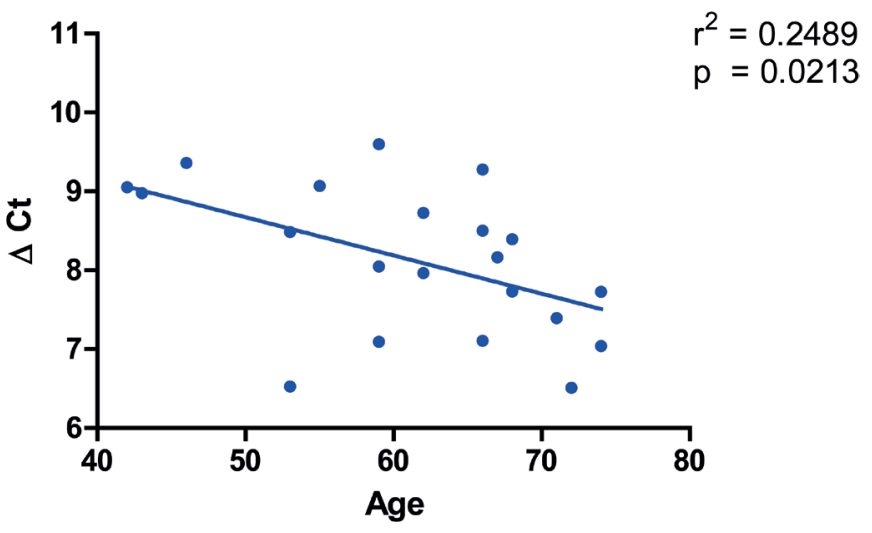

Figure 2 Association between gene expression of EDA2R and age in lung tissue. Age is displayed at the $\mathrm{x}$ axis and $E D A 2 R$ gene expression relative to the housekeeping gene $U 6((\triangle C \mathrm{C})$ on the y axis. project. However, this association was not lung specific, since there were several significant associations in other tissues as well.

Genes differentially expressed with age in patients with COPD compared with non-COPD controls

While our previous analysis resulted in a gene-expression signature for lung ageing and identified $E D A 2 R$ as a strong candidate gene for lung ageing, it did not discriminate between COPD and non-COPD. Since we hypothesise that COPD might be a disease of accelerated lung ageing, we were interested in genes whose expression changed differentially with age between individuals with and without COPD. To find these genes, we next assessed the interaction between age and COPD. An overview of demographical and clinical characteristics of the patients with COPD and non-COPD controls included in this subcohort are presented in online supplementary table S3 of the online supplement.

Our age"COPD interaction analysis identified four DEGs with age in patients with COPD compared with non-COPD controls at an FDR cut-off of $<0.05$. Deoxyribonuclease I-like 3 (DNASE1L3), a gene involved in the complement activation cascade, and retinol binding protein 5 (RBP5), involved in the cellular binding of retinol, were the two DEGs that most significantly increased with age in COPD compared with non-COPD controls. KIAA1462, known to be involved in angiogenesis and blood vessel development, and G protein-coupled 
Table 3 Differentially expressed genes

\begin{tabular}{|c|c|c|c|}
\hline \multicolumn{2}{|l|}{ Genes downregulated with age in COPD } & \multicolumn{2}{|l|}{ Genes upregulated with age in COPD } \\
\hline Name & FDR & Name & FDR \\
\hline KIAA1462 & 0.036 & Deoxyribonuclease I-like 3 (DNASE1L3) & 0.036 \\
\hline Major facilitator superfamily domain containing 4 (MFSD4) & 0.117 & Myosin VIIA and Rab interacting protein (MYRIP) & 0.117 \\
\hline Peptidylglycine alpha-amidating monooxygenase (PAM) & 0.177 & Finkel-Biskis-Reilly murine sarcoma virus (FAU) & 0.127 \\
\hline Inter-alpha-trypsin inhibitor heavy chain family member 5 (ITIH5) & 0.188 & Glioma tumour suppressor candidate region gene 2 (GLTSCR2) & 0.192 \\
\hline CD38 molecule (CD38) & 0.188 & FAM91a2 (FAM91A2) & 0.192 \\
\hline Visinin like 1 (VSNL1) & 0.188 & Haem oxygenase 2 (HMOX2) & 0.192 \\
\hline Intraflagellar transport 57 (IFT57) & 0.192 & Synaptojanin 2 (SYNJ2) & 0.192 \\
\hline Beta-1,3-N-acetylgalactosami-nyltransferase 1 (B3GALNT1) & 0.192 & Sp1 transcription factor (SP1) & 0.193 \\
\hline RAB2A, member RAS oncogene family (RAB2A) & 0.201 & C18orf34 & 0.201 \\
\hline Collagen, type III, alpha 1 (COL3A1) & 0.201 & MYC-associated zinc finger protein (MAZ) & 0.201 \\
\hline Coagulation factor VIII (F8) & 0.201 & Ribosomal protein L38 (RPL38) & 0.201 \\
\hline Solute carrier family 9, subfamily A, member 9 (SLC9A9) & 0.201 & Zinc finger protein 212 (ZNF212) & 0.201 \\
\hline Neurotrophic tyrosine kinase, receptor, type 2 (NTRK2) & 0.201 & ATP binding cassette subfamily $\mathrm{G}$ member 2 (ABCG2) & 0.201 \\
\hline Fucosyltransferase 8 (FUT8) & 0.224 & Phosphoglucomutase 5 pseudogene 1 (PGM5P1) & 0.204 \\
\hline Leucocyte cell derived chemotaxin 1 (LECT1) & 0.224 & Rap guanine nucleotide exchange factor 1 (RAPGEF1) & 0.206 \\
\hline GRINL1A complex locus 1 (GCOM1) & 0.232 & Sodium channel, voltage gated, type III beta subunit (SCN3B) & 0.224 \\
\hline Tumour suppressor candidate 3 (TUSC3) & 0.232 & Protein tyrosine phosphatase, receptor type, R (PTPRR) & 0.224 \\
\hline Solute carrier family 44 , member 1 (SLC44A1) & 0.246 & Calbindin 1 (CALB1) & 0.245 \\
\hline \multirow[t]{10}{*}{ Coiled-coil domain containing 53 (CCDC53) } & 0.248 & Zinc finger protein 764 (ZNF764) & 0.246 \\
\hline & & Leukotriene B4 receptor (LTB4R) & 0.246 \\
\hline & & Nuclear fragile X mental retardation protein interacting protein 2 (NUFIP2) & 0.246 \\
\hline & & Transcription factor-like 5 (TCFL5) & 0.246 \\
\hline & & C7orf20 & 0.246 \\
\hline & & Fibrillarin (FBL) & 0.246 \\
\hline & & hCG (hCG33730) & 0.246 \\
\hline & & Oxidase (cytochrome c) assembly 1-like (OXA1L) & 0.246 \\
\hline & & Nuclear factor I/C (NFIC) & 0.246 \\
\hline & & Sad1 and UNC84 domain containing 2 (UNC84B) & 0.248 \\
\hline
\end{tabular}

receptor 173 (GPR173), involved in tissue homoeostasis, were most significantly decreased with age in patients with COPD compared with non-COPD controls. When using a more lenient FDR cut-off of $<0.25$, we found 100 probe sets coding for 69 genes differentially expressed in association with age in patients with COPD compared with non-COPD controls (table 3).

We decided to use GSEA for the pathway analysis as this is based on the complete ranked gene list reflecting the interaction between age and COPD. GSEA analysis showed that genes that decreased more with age in COPD compared with control were significantly enriched in the extracellular matrix (ECM)-receptor interaction pathway (figure $3 \mathrm{~A}$ ). This indicates that the expression of ECM-related genes is more decreased with age in patients with COPD than in non-COPD controls. Of interest, the three collagen genes COL6A3,COL3A1 and COL4A1 were among the core enriched genes in this ECM-receptor interaction pathway (figure 3A). Genes that increased more with age in patients with COPD compared with non-COPD 

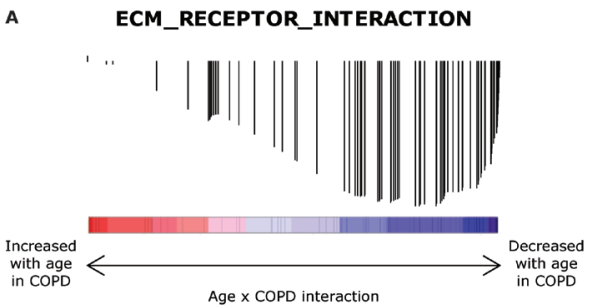

B

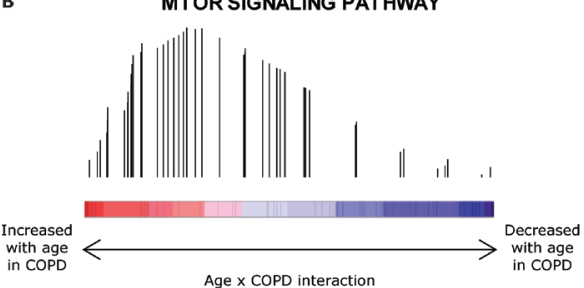

D

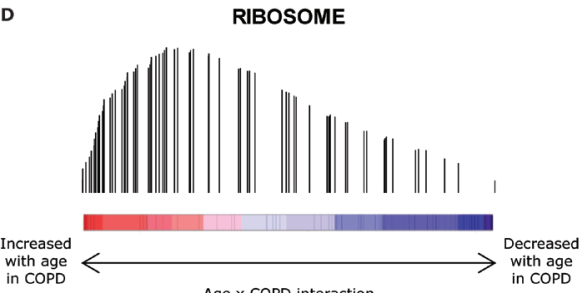

COL6A3
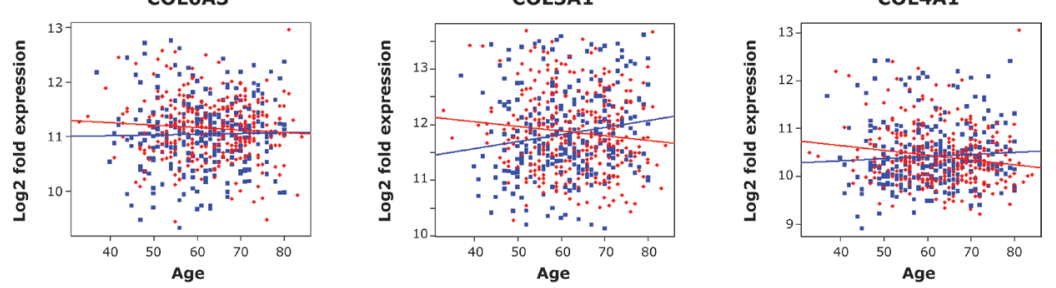

C

SPLICEOSOME
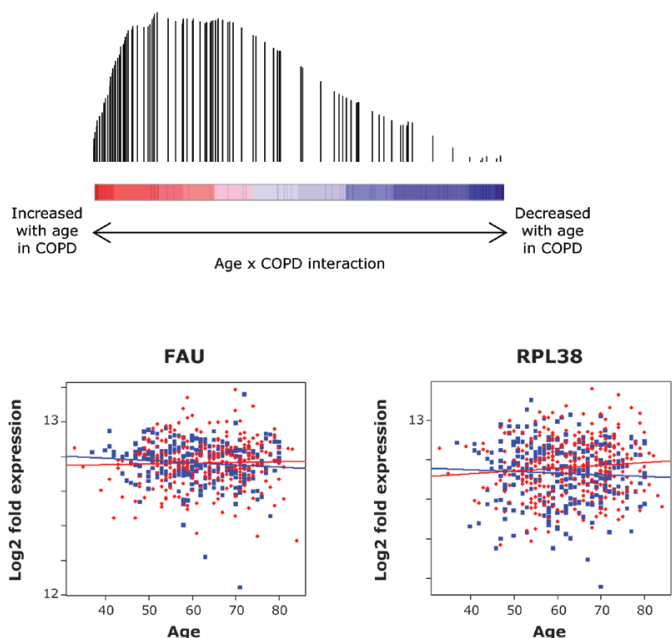

patient with COPD

Figure 3 Pathway enrichment among genes differentially expressed with age between patients with COPD and non-COPD controls. Gene set enrichment analysis shows enrichment of genes whose expression levels significantly change with age in patients with COPD compared with nonCOPD controls. Genes are ranked from left to right based on their expression in the age ${ }^{*}$ COPD interaction analysis in selected patients with COPD and non-COPD controls. The position of each vertical bar indicates the position of a gene whose expression is associated with the indicated pathway within the ranked list. The height of the bar represents the running gene enrichment analysis score. Log2 fold expression of genes differentially expressed with age between patients with COPD and non-COPD controls and core enriched in the subsequent pathway are presented right next to the indicated pathway. Patients with COPD are shown in red and non-COPD controls in blue. ECM receptor interaction pathway with the core enriched genes COL6A3, COL3A1 and COL4A1 was enriched among genes downregulated with age (A). mTOR signalling pathway (B), genes involved in the splicing of introns and exons $(C)$ and genes belonging to the ribosome complex with the core enriched genes FAU and RPL38 (D) were enriched among genes upregulated with age. ECM, extracellular matrix; mTOR, mammalian target of rapamycin.

controls were significantly enriched for the mammalian target of rapamycin (mTOR) signalling pathway (figure 3B). In addition, genes involved in the splicing of introns and exons and genes that belong to the ribosome complex, including the core enriched genes FAU and RPL38, were also more increased with age in patients with COPD compared with non-COPD controls (figure 3C,D, respectively).

\section{Discussion}

In this study, we showed that age strongly affects gene expression in lung tissue. We identified a clear lung tissue gene-expression signature for the ageing lung with 3509 genes being differentially expressed. Genes upregulated with increasing age are enriched in pathways involved in calcium signalling and immune responses, while genes downregulated with increasing age are enriched in pathways involved in lung development and cell-cell contacts. Sensitivity analysis in a subset of patients with COPD and non-COPD controls and in the GTEx project data set demonstrates the robustness of our findings and points towards $E D A 2 R$ as a strong candidate gene for lung ageing. Moreover, the association between $E D A 2 R$ gene expression and age was validated with qRT-PCR in lung tissue. The final analysis in a subset of patients with COPD and non-COPD controls revealed potential roles for genes involved in ECM, mTOR signalling, splicing of introns and exons, and the ribosome complex in abnormal lung ageing in COPD.

Ageing has been a topic of research for decades and with the increasing prevalence of COPD in the elderly, the role of ageing in the pathogenesis of COPD has gained increasing interest. ${ }^{4}$ Recently, Lopez-Otin et al defined nine general hallmarks of ageing, ${ }^{5}$ including genomic instability, telomere attrition and cellular senescence. Several of these hallmarks have also been observed in COPD, ${ }^{79}$ supporting a role of abnormal ageing in COPD. However, most of these studies have been performed in blood leucocytes and data on actual lung tissue is limited. In addition, not much is known yet about the genes underpinning lung ageing in general and accelerated lung ageing in COPD. Therefore, our cross-sectional study on differential gene expression in lung tissue of patients associated with their age is a first attempt to overcome these knowledge gaps. We revealed that processes related to calcium signalling, immune responses, lung development and cell-cell contacts are common between the most significant DEGs in our analysis. The most abundant biological processes among the genes downregulated with age 
comprise development of the respiratory system and cell-cell contacts. The observations on developmental pathways are in line with the theory that ageing might be a result of developmental decay, stating that ageing occurs as a consequence of alterations in developmental pathways. ${ }^{16}$ For pathways related to cell-cell contacts, it has been shown that the permeability of the intestinal epithelium decreases with age in humans ${ }^{17}$ and decreased expression of tight junction molecules has been observed in aged rats. ${ }^{18}$ Interestingly, within our research group reduction of tight junction molecules has been demonstrated in epithelium of patients with COPD, linking this specific hallmark with COPD. ${ }^{19}$ The enriched biological processes among genes upregulated with age involve calcium signalling and immune response. Calcium is an important intracellular signal, functioning in many different cellular processes, ${ }^{20}$ however to our knowledge a role for calcium in COPD has not been described. Ageing, and in particular ageing in COPD, has been associated with immunosenescence (age-related decline in the function of the immune system) and inflammageing (immunosuppression and low-grade inflammation). ${ }^{2122}$ Our results further support the contribution of immune regulatory processes to lung ageing by showing enrichment of these processes at gene-expression level. The sensitivity analyses showed the robustness of our lung ageing gene-expression signature, and revealed a considerably large overlap between our signature and the lung ageing gene-expression signature obtained in the GTEx project. The observation that $E D A 2 R$ was highly significant in all the analyses and the validation of the association between expression of $E D A 2 R$ and age in lung tissue suggest that $E D A 2 R$ might function as a potential biomarker for lung ageing. In contrast to validation of the mRNA expression of EDA2R in lung tissue, it was not possible to reliably quantify EDA2R at the protein level due to weak and unspecific staining on both western blotting and immunohistochemistry. Evaluating the ageing signature of the other tissues from the GTEx project ${ }^{14}$ shows that EDA2R is also highly associated with ageing in adipose tissue, artery, heart, muscle and skin tissue. Therefore, EDA2R seems not to be specific for lung ageing. EDA2R belongs to the tumour necrosis factor receptor superfamily and has been associated with the Nuclear Factor Kappa B (NF-кB) and p53 signalling pathways. ${ }^{23} 24$ The function of $E D A 2 R$ should be further explored before we can make any statement on $E D A 2 R$ as a marker for (lung) ageing.

The second aim of our study was to identify key genes involved in abnormal lung ageing in COPD. Based on our GSEA analysis, we identified four significantly enriched KEGG pathways. The ECM receptor interaction pathway was the only significantly enriched pathway among genes downregulated with increasing age in COPD compared with controls. One of the main phenotypes of COPD is emphysema, in which breakdown of the alveolar walls causes decreased gas exchange in the alveoli. ${ }^{2}$ Loss of ECM, and in particular a lack of repair, plays an important role in emphysema. Our observation that the expression of genes involved in ECM is more decreased with age in patients with COPD compared with non-COPD controls is in line with emphysema development in COPD. Furthermore, three collagen genes COL6A3, COL3A1 and COL4A1 are core enriched in the ECM receptor interaction pathway. Interestingly, the expression of COL6A3 and COL3A1 has been shown to be decreased in aged lungs compared with adult lungs in an ageing mouse model. ${ }^{25}$ Since collagens belong to the main components of $\mathrm{ECM},{ }^{26}$ this further strengthens our observation that ECM modulation plays an important role in abnormal lung ageing in COPD. We found mTOR signalling, ribosome and spliceosome pathways significantly enriched among genes upregulated with age in patients with COPD compared with controls. mTOR is involved in cellular growth and metabolism and the mTOR complex has been established as a central, evolutionary conserved regulator of longevity. ${ }^{27}$ Moreover, it has been shown that inhibition of mTOR can reverse cellular senescence, one of the main hallmarks of ageing. ${ }^{28}$ The fact that one of the upstream activators of mTOR, phosphoinositide-3-kinase, is increased in peripheral blood mononuclear cells of patients with COPD ${ }^{29} 30$ gives interesting leads to further explore in COPD lungs. In contrast to the mTOR signalling pathway, not much is known yet about the specific contribution of the ribosome complex and splicing of introns and exons to COPD and ageing. While protein synthesis (ribosome) and the splicing of introns and exons (spliceosome) are indispensable biological processes, their exact role in accelerated lung ageing in COPD has to be further explored.

Notwithstanding the fact that we identified clear gene-expression signatures for lung ageing and accelerated lung ageing in COPD, we have to consider some limitations of our study. First of all, there are differences between the cohorts with respect to age, smoking status and lung function, which might be attributed to the higher number of patients with early onset, very severe COPD included in the Groningen cohort. ${ }^{31}$ Although we adjusted for intercohort differences by analysing the three cohorts separately followed by a meta-analysis, we cannot completely exclude some cohort-related skewing of the results. Second, the power of our age*COPD interaction analysis was limited as the analysis was performed merely on COPD and non-COPD control subjects for the three centres separately, followed by a meta-analysis. The power limitation is reflected by the relatively low number of significantly DEGs after correction for multiple testing. Nevertheless, our GSEA analysis on the age ${ }^{*}$ COPD interaction takes the complete gene list into account and clearly identified enrichment of pathways relevant to ageing and COPD.

In conclusion, we identified a clear age-related lung tissue gene-expression signature and identified several genes for which a role can be envisaged in abnormal lung ageing in COPD. Based on these findings, we propose processes in control of lung development, cell-cell contacts, calcium signalling and the immune response as important processes in lung ageing in general. Furthermore, we suggest processes in control of ECM homoeostasis, mTOR signalling, splicing of introns and exons, and the ribosome complex to be involved in abnormal lung ageing in COPD.

\section{Author affiliations}

${ }^{1}$ University of Groningen, University Medical Center Groningen, Department of Pathology and Medical Biology, Groningen, The Netherlands

2University of Groningen, University Medical Center Groningen, Department of Epidemiology, Groningen, The Netherlands

${ }^{3}$ University of Groningen, University Medical Center Groningen, Groningen Research Institute for Asthma and COPD, Groningen, The Netherlands

${ }^{4}$ University of Groningen, University Medical Center Groningen, Department of Pulmonary Diseases, Groningen, The Netherlands

${ }^{5}$ European Research Institute for the Biology of Ageing, Universitair Medisch Centrum Groningen, Groningen, Netherlands

${ }^{6}$ Center for Heart Lung Innovation, St Paul's Hospital, The University of British Columbia, Vancouver, Canada

${ }^{7}$ Respiratory Division, University of British Columbia, Vancouver, Canada ${ }^{8}$ Institut Universitaire de Cardiologie et de Pneumologie de Québec, Laval University, Quebec, Canada

${ }^{9}$ Department of Molecular Medicine, Laval University, Quebec, Canada

${ }^{10}$ Merck Research Laboratories, Boston, Massachusetts, USA

Contributors MdV, AF, DSP, DDS, YB, DN, VG, WT, MvdB and C-AB were involved in the conception, design and/or supervision of research; MdV, AF, RRW and TVdJ analysed data; MdV, AF, DSP, WT, MvdB and C-AB interpreted results of analyses; 
MdV prepared figures and drafted the manuscript; AF, DSP, WT, MvdB and C-AB. critically reviewed and revised the manuscript; all authors read and approved the final version of the manuscript. The authors would like to thank Wierd Kooistra for his technical assistance with the EDA2RqRT-PCR validation.

Funding This work was supported by an UMCG Healthy Ageing Pilot grant. CAB is an active member of COST Action BM1201.

Competing interests None declared.

Ethics approval The ethics committees of the Institut Universitaire de Cardiologie et de Pneumologie de Québec, UBC-Providence Health Care Research Ethics Board and University Medical Center Groningen conform the Dutch national ethical and professional guidelines.

Provenance and peer review Not commissioned; externally peer reviewed.

(c) Article author(s) (or their employer(s) unless otherwise stated in the text of the article) 2018. All rights reserved. No commercial use is permitted unless otherwise expressly granted.

\section{REFERENCES}

1 Global Initiative for Chronic Obstructive Lung Disease. From the Global Strategy for the diagnosis, management and prevention of COPD, 2015. http://www.goldcopd. org/

2 Hogg JC, Timens W. The pathology of chronic obstructive pulmonary disease. Annu Rev Pathol 2009;4:435-59.

3 Ito K, Barnes PJ. COPD as a disease of accelerated lung aging. Chest 2009;135:173-80

4 Mercado N, Ito K, Barnes PJ. Accelerated ageing of the lung in COPD: new concepts. Thorax 2015;70:482-9.

5 López-Otín C, Blasco MA, Partridge L, et al. The hallmarks of aging. Cell 2013;153:1194-217

6 Meiners S, Eickelberg 0, Königshoff M. Hallmarks of the ageing lung. Eur Respir J 2015:45:807-27.

7 Savale L, Chaouat A, Bastuji-Garin S, et al. Shortened telomeres in circulating leukocytes of patients with chronic obstructive pulmonary disease. Am J Respir Crit Care Med 2009;179:566-71.

8 Albrecht E, Sillanpaa E, Karrasch S, et al. Telomere length in circulating leukocytes is associated with lung function and disease. Eur Respir J 2014;43:983-92.

9 Rutten EP, Gopal P, Wouters EF, et al. Various mechanistic pathways representing the ageing process are altered in COPD. Chest 2015

10 Kanaji N, Basmaé $\mathrm{H}$, Nelson A, et al. Fibroblasts that resist cigarette smoke-induced senescence acquire profibrotic phenotypes. Am J Physiol Lung Cell Mol Physiol 2014:307:L364-L373.

11 Hao K, Bossé Y, Nickle DC, et al. Correction: Lung eQTLs to Help Reveal the Molecular Underpinnings of Asthma. PLoS Genet 2012;8.

12 Brandsma CA, van den Berge M, Postma DS, et al. A large lung gene expression study identifying fibulin-5 as a novel player in tissue repair in COPD. Thorax $2015 ; 70: 21-32$
13 R Development Core Team. R: a language and environment for statistical computing, 2011. ISBN:3-900051-07-0.

14 Yang J, Huang T, Petralia F, et al. Synchronized age-related gene expression changes across multiple tissues in human and the link to complex diseases. Sci Rep 2015;5:15145

15 Fehrmann RS, Karjalainen JM, Krajewska M, et al. Gene expression analysis identifies global gene dosage sensitivity in cancer. Nat Genet 2015;47:115-25.

16 Martin N, Beach D, Gil J. Ageing as developmental decay: insights from p16(INK4a.). Trends Mol Med 2014;20:667-74.

17 Man AL, Bertelli E, Rentini S, et al. Age-associated modifications of intestinal permeability and innate immunity in human small intestine. Clin Sci 2015:129:515-27.

18 Ren WY, Wu KF, Li X, et al. Age-related changes in small intestinal mucosa epithelium architecture and epithelial tight junction in rat models. Aging Clin Exp Res 2014;26:183-91.

19 Heijink IH, Noordhoek JA, Timens W, et al. Abnormalities in airway epithelial junction formation in chronic obstructive pulmonary disease. Am J Respir Crit Care Med 2014:189:1439-42.

20 Berridge MJ, Bootman MD, Roderick HL. Calcium signalling: dynamics, homeostasis and remodelling. Nat Rev Mol Cell Biol 2003:4:517-29.

21 Murray MA, Chotirmall SH. The impact of immunosenescence on pulmonary disease. Mediators Inflamm 2015;2015:1-10.

22 Sharma G, Hanania NA, Shim YM. The aging immune system and its relationship to the development of chronic obstructive pulmonary disease. Proc Am Thorac Soc 2009:6:573-80.

23 Verhelst K, Gardam S, Borghi A, et al. XEDAR activates the non-canonical NF- $\kappa B$ pathway. Biochem Biophys Res Commun 2015;465:275-80.

24 Tanikawa C, Furukawa Y, Yoshida N, et al. XEDAR as a putative colorectal tumor suppressor that mediates p53-regulated anoikis pathway. Oncogene 2009;28:3081-92.

25 Williams AE, Perry MM, Moschos SA, et al. microRNA expression in the aging mouse lung. BMC Genomics 2007:8:172.

26 Humphrey JD, Dufresne ER, Schwartz MA. Mechanotransduction and extracellular matrix homeostasis. Nat Rev Mol Cell Biol 2014;15:802-12.

27 Johnson SC, Rabinovitch PS, Kaeberlein M. mTOR is a key modulator of ageing and age-related disease. Nature 2013;493:338-45

28 Walters HE, Deneka-Hannemann S, Cox LS. Reversal of phenotypes of cellular senescence by pan-mTOR inhibition. Aging 2016;8:231-44.

29 To Y, Ito K, Kizawa Y, et al. Targeting phosphoinositide-3-kinase-delta with theophylline reverses corticosteroid insensitivity in chronic obstructive pulmonary disease. Am J Respir Crit Care Med 2010;182:897-904.

30 Barnes PJ. Mechanisms of development of multimorbidity in the elderly. Eur Respir J 2015:45:790-806

31 Silverman EK, Chapman HA, Drazen JM, et al. Genetic epidemiology of severe, early-onset chronic obstructive pulmonary disease. Risk to relatives for airflow obstruction and chronic bronchitis. Am J Respir Crit Care Med 1998;157:1770-8 